\title{
Do Transfusions Cause Necrotizing Enterocolitis? Evidence and Potential Mechanisms
}

\author{
Edmund F. LaGamma ${ }^{1,2}$
}

$\mathbf{T}$ RANSFUSION-ASSOCIATED GUT INJURY (TRAGI) refers to the temporal association ( $<48$ hours) between packed red blood cell (PRBC) transfusion and the development of necrotizing enterocolitis (NEC) in very low birth weight neonates (Fig. 1). ${ }^{1}$ Other than extreme prematurity, TRAGI cases lack many of the classical risk factors associated with NEC (e.g., low Apgar scores, hypotension, hypoxia, umbilical catheters, feeding intolerance, and sepsis). A comparison of several recent reports from different neonatal intensive care units (NICUs) showed TRAGI accounted for $\sim 30 \%$ of all NEC cases. TRAGI was associated with lower gestational age $(<28$ weeks), lower birth weight $(<1,000 \mathrm{~g})$, extreme anemia (hematocrit [Hct] $\leq 25 \%$ ), and transfusion of older stored blood ( $>10$ days). Despite the association between PRBC transfusion and NEC, there is limited evidence (i.e., a lack of randomized clinical trial data, particularly regarding temporality) to support a causal relationship. ${ }^{2,3}$

A retrospective chart review of 65 infants in the NICU who developed NEC found that formula-fed infants accounted for $72 \%(n=47 / 65)$ of NEC cases, whereas only $28 \%(n=18 / 65)$ of infants who received breast milk developed NEC. ${ }^{4}$ These findings suggest that breast milk is protective against NEC; however, the incidence of TRAGI was similar in the infants who received formula and those who received breast milk ( $32 \%$ and $28 \%$, respectively), suggesting that breast milk does not protect against TRAGI. Therefore, TRAGI likely originates on the vascular side, as opposed to the luminal side, of the mucosal barrier.

Extreme anemia $(\mathrm{Hct}<25 \%)$ has been associated with increased risk of NEC after PRBC transfusion in preterm infants. ${ }^{5}$ PRBC transfusions also cause a failure of physiologic reflex responses to enteral feeding in anemic very low birth weight infants. ${ }^{6}$ Enteral feeds increase gut motility and digestion and consequently oxygen consumption; thus, they may disrupt the balance of oxygen delivery and consumption. Physiologic mechanisms to compensate for extreme anemia include increased cardiac output, increased oxygen extraction due to reduced oxygen delivery, elevated erythropoietin levels, and release of hemoglobin-bound nitric oxide. ${ }^{7}$ These processes may result in a reaction to $\mathrm{PRBC}$ products due to factors associated with red blood cell (RBC) storage lesion (Table 1$){ }^{8}$ Of these factors, only the hypoxic vasodilation effect becomes physiologically significant during extreme anemia. RBC storage lowers RBC nitric oxide and increases nitric oxide scavenging by free hemoglobin, which has a 100- to 1,000-fold higher affinity for nitric oxide than

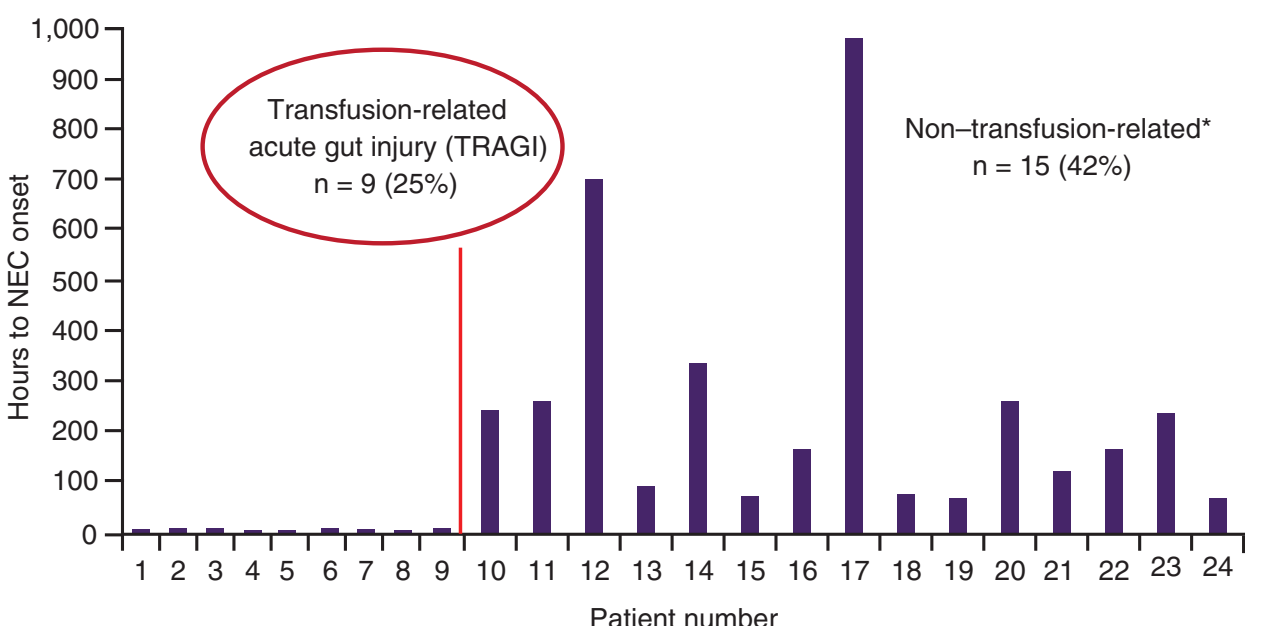

FIG. 1. Number of hours to onset of NEC after PRBC transfusion. ${ }^{1} * 12(33 \%)$ additional subjects had NEC but no prior transfusion. NEC, necrotizing enterocolitis; PRBC, packed red blood cell. Reprinted from The Journal of Pediatrics, Vol.158, No. 3, Blau J et al., Transfusion-Related Acute Gut Injury: Necrotizing Enterocolitis in Very Low Birth Weight Neonates after Packed Red Blood Cell Transfusion, pp. 403-409, Copyright (C) 2011, with permission from Elsevier.

\footnotetext{
${ }^{1}$ Professor of Pediatrics, Biochemistry, and Molecular Biology, Chief of the Division of Newborn Medicine, and Director of the Neonatal-Perinatal Fellowship Program, New York Medical College, Valhalla, New York.

${ }^{2}$ Chief of the Regional Neonatal Intensive Care Unit (NICU) of Maria Fareri Children's Hospital at Westchester Medical Center, Valhalla, New York.
} 
Table 1. Factors Associated with Red Blood Cell Storage Lesion

(1) RBC physical and structural-increased propensity to obstruct flow or cause vasoconstriction

- Stacking (e.g., "roll of coins"); microaggregates (flow obstruction)

- Increased adhesiveness ("stickiness")

- Stiffer, less deformable (difficulty in passing through capillaries)

- Increased viscosity due to higher Hct

- RBC fragmentation creating microparticles $\mathrm{w} / \mathrm{Hgb}$ and free $\mathrm{Hgb}$ (NO scavenging)

- Free membrane lipids (vasoactive)

(2) RBC metabolic-altered $\mathrm{O}_{2}$ carrying capacity; vascular effects

- Low $\mathrm{pO}_{2}$ plus low 2,3 DPG (higher affinity for $\mathrm{O}_{2}$-less $\mathrm{O}_{2}$ available)

- Increased lactate, low pH, large base deficit, higher $\mathrm{K}+$ (altered vascular resistance)

- Lower ATP (RBC energy crisis)

- Donor-specific reduced survivability (cysteine, caffeine, etc.)

- Free iron (oxidant injury)

(3) Nitric oxide-increased propensity to cause vasoconstriction due to NO depletion

- Reduced RBC NO, S-NO-Hgb during storage

- Lower pH+derivative left shift in $\mathrm{P}_{50}$ of RBC

- $\mathrm{P}_{50}$ shift depletes nitrite substrate through oxy-Hgb converting it to nitrate

- Free Hgb has 100-1,000-fold higher affinity for NO than RBC Hgb

(4) Humoral factors-host versus graft reactions; inflammatory mediators released

- Haplotype antibodies (anti-HLA in second pregnancy)

- Cytokines released from WBC during filtering to remove CMV/WBC

- Reactive increase in PAF from infused humoral factors

- Activation of endothelial receptors by humoral factors

- T-antigen exposure

Factors associated with red blood cell storage lesion. ${ }^{8}$

ATP, adenosine triphosphate; CMV, cytomegalovirus; DPG, diphosphoglycerate; Hgb, hemoglobin; HLA, human leukocyte antigen; $\mathrm{NO}$, nitric oxide; $\mathrm{pO}_{2}$; oxygen partial pressure; $\mathrm{P}_{50}$, partial pressure of a gas required to achieve $50 \%$ hemoglobin saturation; $\mathrm{PAF}$, platelet activating factor; RBC, red blood cell; S-NO-Hgb, S-nitrosylated hemoglobin; WBCs, white blood cells.

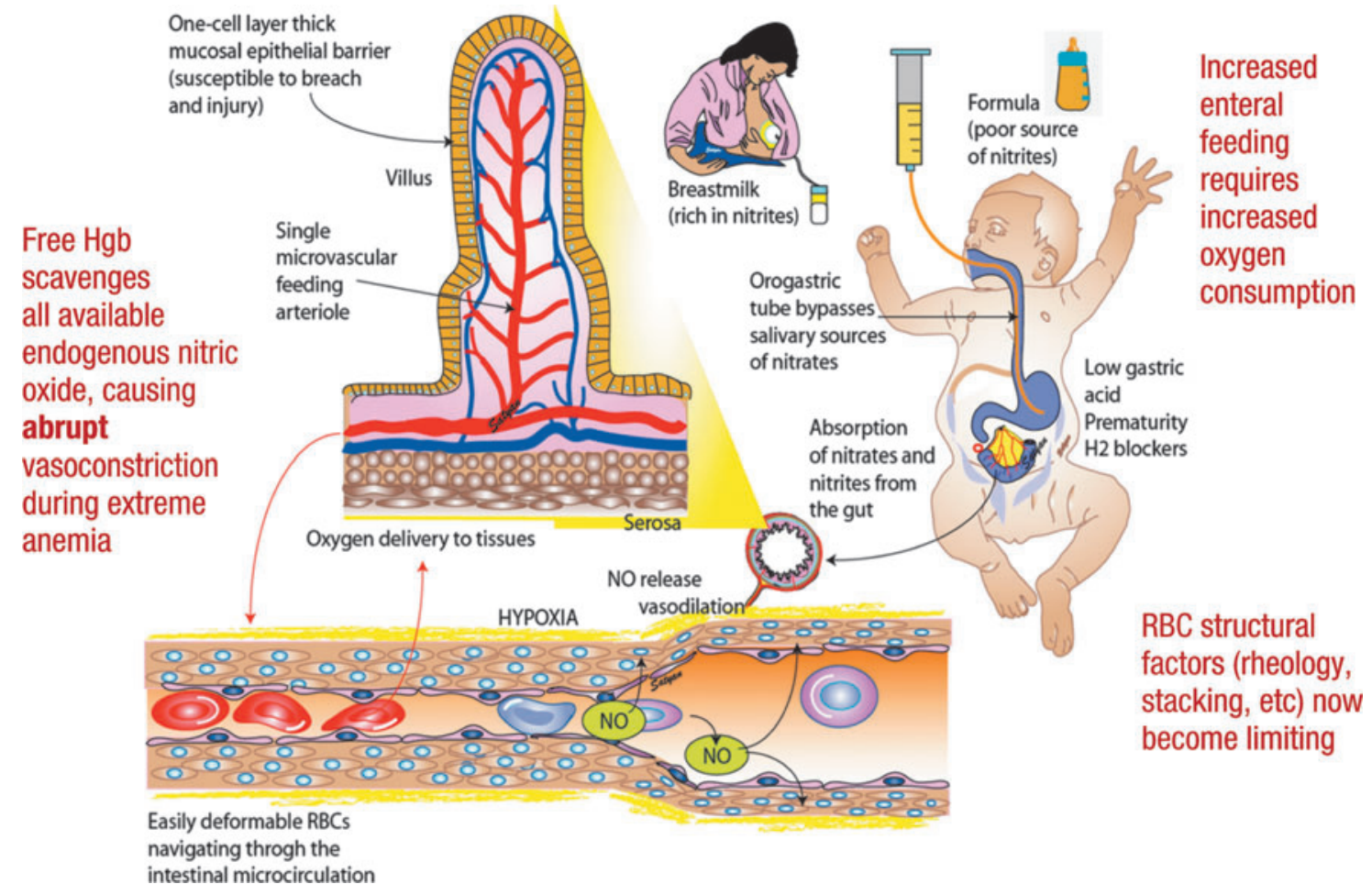

FIG. 2. Proposed mechanism for TRAGI ${ }^{8} \mathrm{Hgb}$, hemoglobin; NO, nitric oxide; TRAGI, transfusion-associated gut injury. Reproduced with permission from NeoReviews, Vol. 16, No. 7, pp. e420-e430, Copyright (C) 2015, by the AAP. 
RBCs containing hemoglobin. Nitric oxide scavenging after transfusion can result in perturbations in nitric oxidedependent hypoxic vasodilation and reduced blood flow. ${ }^{8,9}$ Unique arteriole branching in the intestinal villi makes these structures more susceptible to conditions that cause oxygen deprivation (e.g., anemia and flow disturbances). ${ }^{10}$ In addition, RBC storage causes physical and structural changes in RBCs (e.g., changes in rheology, increased adhesiveness, more stiffness, and less malleable), which may cause injury to the intestinal microvasculature. ${ }^{8}$ Thus, RBC storage lesions in combination with other risk factors may contribute to the development of TRAGI. Increased oxygen demand (enteral feeding) in the setting of extreme anemia and PRBC transfusion may result in injury to the mucosal barrier, enabling bacterial invasion and NEC (Fig. 2). ${ }^{8,11}$

Inhaled nitric oxide has been shown to improve systemic microcirculation in infants and children with hypoxemic respiratory failure. ${ }^{12}$ It is possible that inhaled nitric oxide could be used to prevent nitric oxide scavenging by free hemoglobin when giving PRBC transfusions for extreme anemia. A clinical trial (iNO-TRAGI) is currently underway to assess whether providing inhaled nitric oxide during and after a PRBC transfusion for anemia can improve oxygen delivery and prevent TRAGI in preterm infants.

\section{References}

1. Blau J, Calo JM, Dozor D, et al. Transfusion-related acute gut injury: Necrotizing enterocolitis in very low birth weight neonates after packed red blood cell transfusion. J Pediatr 2011;158:403-409.

2. Kirpalani H, Zupancic JA. Do transfusions cause necrotizing enterocolitis? The complementary role of randomized trials and observational studies. Semin Perinatol 2012; 36:269-276.

3. Hay S, Zupancic JA, Flannery DD, et al. Should we believe in transfusion-associated enterocolitis? Applying a GRADE to the literature. Semin Perinatol 2017;41:80-91.

4. Shillingford K. Protective effects of breast milk fail to prevent transfusion related acute gut injury (TRAGI). Poster presented at Pediatric Academic Societies and Asian
Society for Pediatric Research 2014 Joint Meeting, Vancouver, Canada, May 3-6, 2014.

5. Singh R, Visintainer PF, Frantz ID 3rd, et al. Association of necrotizing enterocolitis with anemia and packed red blood cell transfusions in preterm infants. J Perinatol 2011;31: 176-182.

6. Krimmel GA, Baker R, Yanowitz TD. Blood transfusion alters the superior mesenteric artery blood flow velocity response to feeding in premature infants. Am J Perinatol 2009;26:99-105.

7. Alkalay AL, Galvis S, Ferry DA, et al. Hemodynamic changes in anemic premature infants: Are we allowing the hematocrits to fall too low? Pediatrics 2003;112:838-845.

8. La Gamma EF, Feldman A, Mintzer J, et al. Red blood cell storage in transfusion-related acute gut injury. NeoReviews 2015;16:e420-e430.

9. Vermeulen Windsant IC, de Wit NC, Sertorio JT, et al. Blood transfusions increase circulating plasma free hemoglobin levels and plasma nitric oxide consumption: A prospective observational pilot study. Crit Care 2012; 16:R95.

10. Nowicki PT. Ischemia and necrotizing enterocolitis: Where, when, and how. Semin Pediatr Surg 2005;14: $152-158$.

11. La Gamma EF, Browne LE. Feeding practices for infants weighing less than $1500 \mathrm{G}$ at birth and the pathogenesis of necrotizing enterocolitis. Clin Perinatol 1994;21:271-306.

12. Top AP, Ince $\mathrm{C}$, Schouwenberg $\mathrm{PH}$, et al. Inhaled nitric oxide improves systemic microcirculation in infants with hypoxemic respiratory failure. Pediatr Crit Care Med 2011; 12:e271-e274.

Address correspondence to: Edmund F. LaGamma, MD, FAAP The Regional Neonatal Intensive Care Unit Maria Fareri Children's Hospital at Westchester Medical Center New York Medical College 100 Woods Road Valhalla, NY 10595

E-mail: edmund_lagamma@nymc.edu 